\title{
Adsorção de atrazina por biocarvão de palha de milho ativado em colunas de solo
}

\author{
Adsorption of atrazine for biochar corn straw in soil columns \\ Adsorción de atrazina por biocarbón de paja de maíz activada en columnas de suelo
}

Recebido: 04/11/2021 | Revisado: 12/11/2021 | Aceito: 19/11/2021 | Publicado: 28/11/2021

\author{
Camila Jussara Schmidt \\ ORCID: https://orcid.org/0000-0003-3825-3567 \\ Universidade Federal do Paraná, Brasil \\ E-mail: camilajschmidt@gmail.com \\ Jonathan Dieter \\ ORCID: https://orcid.org/0000-0002-3674-4955 \\ Universidade Federal do Paraná, Brasil \\ E-mail: jony.dieter@gmail.com \\ Silvio César Sampaio \\ ORCID: https://orcid.org/0000-0002-9034-432X \\ Universidade Estadual do Oeste do Paraná, Brasil \\ E-mail: silvio.sampaio@unioeste.br \\ Danielle Medina Rosa \\ ORCID: https://orcid.org/0000-0003-3222-4474 \\ Universidade Estadual do Oeste do Paraná, Brasil \\ E-mail: danimrosa@yahoo.com.br \\ Mariana Sbizzaro \\ ORCID: https://orcid.org/0000-0003-1292-7535 \\ Universidade Estadual do Oeste do Paraná, Brasil \\ E-mail: msbizzaro@hotmail.com \\ Floriano Luiz Suszek \\ ORCID: https://orcid.org/0000-0002-7981-1572 \\ Universidade Estadual do Oeste do Paraná, Brasil \\ E-mail: flsuszek@hotmail.com \\ Adir Otto Schmidt \\ ORCID: https://orcid.org/0000-0002-8282-4139 \\ Universidade Estadual do Oeste do Paraná, Brasil \\ E-mail: adiroschmidt@gmail.com
}

\begin{abstract}
Resumo
A atrazina é um herbicida amplamente utilizado em controle de infestações predominantes de folhas largas em culturas agrícolas como milho, sorgo e cana de açúcar, com alta mobilidade no solo, podendo tornar-se tóxico para os organismos aquáticos, plantas e seres humanos. Assim, a adsorção de pesticidas por biocarvões se torna opção viável, mitigando os impactos ambientais causados. Utilizados em modelos físico-matemáticos, ensaios de lixiviação de íons em coluna de solo, possibilitam a caracterização da mobilidade dos solutos apresentando soluções de equação diferencial de transporte de solutos no solo. Desta forma, o objetivo deste trabalho foi verificar o potencial de adsorção de atrazina por biocarvão de palha de milho em coluna de solo indeformado e deformado. Para isto, o experimento foi conduzido em Delineamento Inteiramente Casualizado (DIC), totalizando 16 ensaios (coluna deformada com biocarvão, coluna deformada sem biocarvão, coluna indeformada com biocarvão e coluna indeformada sem biocarvão). Os tratamentos com biocarvão receberam uma quantidade de 5 ton ha ${ }^{-1}$ e todos receberam a mesma quantidade de atrazina $\left(6,5 \mathrm{~L} \mathrm{ha}^{-1}\right)$. O biocarvão foi caracterizado por meio de avaliação da morfologia das superfícies por Microscopia Eletrônica de Varredura (MEV), área superficial específica (ASEBET) e porosidade. As concentrações de atrazina foram medidas por Cromatografia Líquida de Alta Performance (HPLC). No solo indeformado verificou-se diferença significativa na adsorção de atrazina após a aplicação do biocarvão, uma vez que reduziu a mobilidade do pesticida no solo. Porém, o método utilizado no solo deformado não se mostrou eficiente, fazendo-se necessário mais estudos voltados à essa perspectiva.
\end{abstract}

Palavras-chave: Poluição do solo; Curvas de eluição; Herbicida; Triazinas.

\begin{abstract}
Atrazine is a herbicide widely used in control of predominant broad leaf infestations in agricultural crops such as corn, sorgo and sugarcane, with high mobility in the soil, and may become toxic to aquatic organisms, plants and humans. Thus, the adsorption of pesticides by biocoals becomes a viable option, mitigating the environmental impacts caused. Used in physico-mathematical models, ion leaching tests in soil column, allow the characterization of the mobility of solutes presenting differential equation solutions for transportof solutes in the soil. Thus, the present study aims to verify the adsorption potential of atrazine by biochar synthesized from corn straw in an undefined and deformed soil
\end{abstract}


column. For this, the experiment was conducted in a completely randomized design, totaling 16 tests (deformed column with biochar, deformed column without biochar, undisturbed column with biochar and undisturbed column without biochar). The treatments with bio-carbon received an amount of 5 ton $\mathrm{ha}^{-1}$ and all received the same amount of atrazine $\left(6,5 \mathrm{~L} \mathrm{ha}^{-1}\right)$. The biochar was characterized based on their physical and chemical properties, by surface morphology evaluation (Scanning Electron Microscopy - SEM), specific surface area and porosity. Atrazine concentrations were measured by High Performance Liquid Chromatography (HPLC). In the undisturbed soil, it was found that significant differences in adsorption of atrazine after application of the biochar, once reduced pesticide mobility in soil. However, the method used in soil column deformed was not efficient, making necessary more studies to this perspective.

Keywords: Soil pollution; Elution curves; Herbicide; Triazines.

\section{Resumen}

La atrazina es un herbicida ampliamente utilizado en el control de infestaciones predominantes de hojas anchas en cultivos agrícolas como el maíz, el sorgo y la caña de azúcar, con alta movilidad en el suelo, y puede llegar a ser tóxico para los organismos acuáticos, las plantas y los seres humanos. Así, la adsorción de plaguicidas por biocarbones se convierte en una opción viable, mitigando los impactos ambientales causados. Utilizados en modelos físico-matemáticos, los ensayos de lixiviación iónica en columna de suelo, permiten la caracterización de la movilidad de solutos presentando soluciones de ecuaciones diferenciales para el transporte de solutos en el suelo. Así, el objetivo de este trabajo fue verificar el potencial de adsorción de atrazina por el biocarbón de paja de maíz en una columna de suelo no deformada y deformada. Para ello, el experimento se realizó en un Diseño Completamente Aleatorizado (IHD), totalizando 16 ensayos (columna deformada con biocarbón, columna deformada sin biocarbón, columna indeformada con biocarbón y columna no deformada sin biocarbón). Los tratamientos de biocarbón recibieron una cantidad de 5 toneladas ha-1 y todos recibieron la misma cantidad de atrazina (6,5 L ha-1). El biocarbón se caracterizó por evaluar la morfología de las superficies mediante Microscopía Electrónica de Barrido, área de superficie específica (ASEBET) y porosidad. Las concentraciones de atrazina fueron determinados por cromatografía líquida de alto rendimiento (HPLC). En el suelo no deformado, hubo una diferencia significativa en la adsorción de atrazina después de la aplicación del biocarbón, ya que redujo la movilidad del pesticida en el suelo. Sin embargo, el método utilizado en el suelo deformado no fue eficiente, por lo que los estudios posteriores se centraron en esta perspectiva.

Palabras clave: Contaminación del suelo; Curvas de elución; Herbicida; Trianais.

\section{Introdução}

A atrazina é um herbicida de ação seletiva pré e pós-emergente, utilizado em controle de infestações predominantes de folhas largas e presença de gramíneas sensíveis, em culturas agrícolas de folhas estreitas, como milho, sorgo e cana de açúcar (Borras et al., 2010). Possui potencial de contaminação de águas subterrâneas, apresentando médio potencial de contaminação em função do transporte associado ao sedimento e alto potencial quando dissolvido em água, caracterizada como potencial lixiviador (Barreto, 2006; Canuto et al., 2010). É um herbicida considerado tóxico para os organismos aquáticos, plantas e seres humanos, necessitando técnicas de remoção do solo para atender requisitos de qualidade ambiental (Rojas et al., 2015).

O comportamento de herbicidas no solo é governado pelos processos sortivos que afetam a eficácia destes produtos, e a presença de materiais orgânicos como biocarvão é capaz de aumentar a adsortividade de pesticidas no solo, devido à sua elevada área superficial específica e alta microporosidade (Oliveira Jr., 2002; Alonso et al., 2011; Farias et al., 2016). Pesquisas apontam que biocarvão adsorve efetivamente a atrazina, uma vez que esta é particionada em sua fase orgânica, sendo hidrolisada mais rapidamente na presença de biocarvão (Cao et al., 2009; 2011). Além disso, a afinidade do biocarvão pela atrazina aumenta com a diminuição da relação sólido/solução, assim como a sorção do biocarvão para o herbicida é favorecida pelo baixo pH da solução (Zhang et al., 2013).

Proveniente do processo de pirólise da matéria orgânica em condições de pouca ou nenhuma presença de oxigênio, o biocarvão é um produto rico em carbono (Adams et al., 2011), que segundo a EMBRAPA (2017), é um condicionador que, agregado ao solo, ajuda a melhorar suas características químicas, físicas e biológicas. Além disso, pode ser obtido a partir de diferentes matérias-primas, tais como resíduos agroindustriais, de modo a destinar o passivo ambiental de forma correta. Para que seja considerado bom adsorvente e condicionante de solo, o biocarvão deve apresentar estrutura interna inerte, que faz 
preservar o carbono no solo por muitos anos, e estrutura periférica reativa, para atuar como a matéria orgânica natural do ambiente (Rezende et al., 2011). Pesquisadores concluíram que o biocarvão pode ainda ser utilizado como um mecanismo para a mitigação das mudanças climáticas, melhorando a produção de alimentos, a reabilitação de terras degradadas e a gestão de resíduos (Leach et al., 2012).

O biocarvão pode reduzir a lixiviação de alguns pesticidas em colunas de solo deformadas (Tatarková et al., 2013), porém, o impacto potencial na lixiviação de agrotóxicos em condições de campo não é claro, uma vez que há interferência de fatores como a estrutura de macroporos do solo e o transporte facilitado por colóides que podem influenciar a lixiviação (Cabrera et al., 2011). Entretanto, em solos indeformados, alguns autores observaram que o impacto do biocarvão na lixiviação de vários agroquímicos depende do tipo de solo (Larsbo et al., 2013).

Ensaios de lixiviação de íons em coluna de solo, possibilitam a caracterização da mobilidade dos solutos. Utilizados em modelos físico-matemáticos, esses dados apresentam soluções de equação diferencial de transporte de solutos no solo. Os experimentos podem ser realizados em condições de laboratório ou de campo, e os resultados obtidos com base nos processos de dispersão-difusão e no retardamento do avanço dos solutos em relação ao avanço da interface entre o líquido deslocador e o deslocado (Ferreira, 2007). Em coluna de solo os experimentos demonstraram que o impacto do biocarvão na lixiviação de atrazina é limitado, muitas vezes, associado à eventos de pico de lixiviação, ocorrendo logo após a saturação do solo, e as maiores diferenças na lixiviação de atrazina são observadas durante este pico, com diferenças diminuindo à medida que a lixiviação continua. Isto indica que as reduções a longo prazo na lixiviação de atrazina em água subterrânea são incertas (Delwiche et al., 2014).

Dessa forma, este trabalho avaliou a capacidade de adsorção de atrazina por biocarvão de palha de milho em colunas contendo solo deformado e indeformado, utilizando metodologia experimental e obtendo os parâmetros de transporte do movimento da atrazina no perfil do solo.

\section{Metodologia}

Os tratamentos utilizados foram coluna de solo indeformada com biocarvão (IC), indeformada sem biocarvão (IS), coluna de solo deformada com biocarvão (DC) e deformada sem biocarvão (DS), em quatro repetições. A umidade do solo foi ajustada para capacidade de campo e então o biocarvão foi adicionado na superfície da coluna na concentração de $4 \mathrm{~g}$ coluna

${ }^{1}$, equivalente a aproximadamente 5 ton $\mathrm{ha}^{-1}$ (quando o tratamento consistia na aplicação do produto), e posteriormente foi adicionado o pesticida, na concentração de 6,5 ppm, equivalente a 6,5 $\mathrm{L} \mathrm{ha}^{-1}$.

As colunas foram saturadas com 24 horas de antecedência em água deionizada e posteriormente fixadas verticalmente em uma estrutura de madeira. As colunas foram submetidas à aplicação de água destilada até atingir fluxo constante de saída da coluna (steady state), utilizando-se do permeâmetro de carga constante, com quatro centímetros de carga hidráulica, aproximadamente.

A quantidade total aplicada da solução de atrazina em cada coluna corresponde a cerca de 5,0 volumes de poros. Este volume de poros utilizado para a obtenção dos pontos da curva de efluente foi obtido com base no volume da amostra, nas massas específicas do solo e de partículas e na porosidade total. As amostras de percolado foram coletadas a cada 0,2 volumes de poros, correspondendo a 25 amostras por coluna, sendo as coletas feitas com auxílio de um coletor de frações automático.

Os tempos de coleta foram variados, pois dependiam do fluxo de aplicação e da condutividade hidráulica dos solos, mesmo sendo coletadas amostras com o mesmo volume. Para estimar os tempos de coletas, testes de condutividade hidráulica foram realizados para manter a carga hidráulica na superfície do solo constante. Ao atingir o regime estacionário do fluxo, manteve-se uma lâmina constante acima do solo iguais. Das amostras coletadas, foram construídas curvas de eluição para avaliação do comportamento do pesticida aplicado no solo com e sem biocarvão. 
As colunas foram coletadas em solo manejado sob sistema de semeadura direta. A coleta das colunas indeformadas foi realizada com auxílio de tubos para irrigação de PVC, com $40 \mathrm{~cm}$ de altura e diâmetro interno de $10 \mathrm{~cm}$. Antes da coleta, o solo foi submetido a um pré-molhamento. Esta saturação do solo com água deixou-o na consistência de fluidez, facilitando assim, a introdução do tubo e preservando a estrutura do solo coletado. Nessas condições, evita-se a formação de fissuras no interior da coluna pelo efeito de um eventual atrito com a parede interna durante a descida deste para o interior do solo. Posteriormente, os tubos foram introduzidos no sentido vertical, sendo necessário a utilização de uma marreta e sequentes molhamentos externos, e depois, utilizado macaco hidráulico sob um trator. O controle da profundidade foi feito com uma marcação aos $30 \mathrm{~cm}$ de altura nos tubos.

A retirada dos tubos do interior do solo ocorreu de forma manual com o auxílio de uma pá, para evitar a deformação do solo no interior do tubo. Para finalizar a coleta das colunas, o solo aderido à parede externa do tubo foi retirado, bem como o excesso de solo existente na parte inferior, fazendo com que este ficasse no mesmo plano da extremidade inferior do tubo. A fim de evitar que a coluna de solo se deslocasse para baixo, foi colocada uma manta sintética, devidamente presa no tubo com abraçadeiras metálicas na extremidade inferior do tubo.

A preparação das colunas deformadas foi baseada na metodologia da ISO 11464 (1994), isto é, o solo foi destorroado, secado ao ar e posteriormente passado em peneira de malha 2,0 $\mathrm{mm}$. A quantidade de solo em cada coluna foi calculada a partir da densidade do solo, analisada em laboratório, e de acordo com o volume determinado para a coluna. As colunas se assemelharam às condições reais do solo analisado, principalmente na uniformização do volume de poros nas colunas. Após pesado, o solo foi colocado nas colunas, de forma que a quantidade de solo calculada atingisse a altura de $30 \mathrm{~cm}$ na coluna.

O biocarvão ativado foi produzido a partir da palha de milho. As amostras de palha de milho in natura foram moídas e peneiradas em uma peneira com malha de $0,177 \mathrm{~mm}$, para posterior ativação química. Para a ativação química da palha de milho, misturou-se aproximadamente 10 gramas da biomassa à $100 \mathrm{~mL}$ de solução ativante - $\mathrm{H}_{3} \mathrm{PO}_{4}$ (Razão mássica 1:10) na concentração de $1,5 \mathrm{~mol} \mathrm{~L}^{-1}$, encaminhou-se a mistura ao agitador por duas horas na rotação de $100 \mathrm{rpm}$ e temperatura de $25^{\circ} \mathrm{C}$. Posteriormente, a amostra foi filtrada em sistema a vácuo, e encaminhada a estufa por 24 horas à $105^{\circ} \mathrm{C}$. Após a etapa de ativação, realizou-se a pirolise da amostra, sob baixa concentração de oxigênio, à temperatura de $400{ }^{\circ} \mathrm{C}$, com taxa de aquecimento de $30^{\circ} \mathrm{C} \mathrm{min}^{-1}$, durante o período de seis horas. Para finalizar, foi realizada a lavagem do biocarvão ativado com água destilada, até o pH atingir a neutralidade, para remoção de $\mathrm{H}_{3} \mathrm{PO}_{4}$ residual. Então, o biocarvão ativado foi seco em estufa à $105^{\circ} \mathrm{C}$, durante 24 horas, macerado, peneirado à $0,075 \mathrm{~mm}$, para assim, utilizá-lo nos ensaios experimentais.

Caracterizado química e fisicamente, o biocarvão foi avaliado por Microscopia Eletrônica de Varredura (MEV) e área superficial, diâmetro de poro, volume total de poro e volume de microporos pelo método Brunauer-Emmett-Teller (BET). Para a Microscopia Eletrônica de Varredura (MEV), a amostra foi colocada sobre fita de carbono dupla face, seca e metalizada com uma fina camada (5nm) de ouro na superfície (sputtering). As micrografias foram obtidas nos aumentos de $7 \mathrm{Kx}$ e $10 \mathrm{Kx}$, em módulo SE (Secondary Electron) com detector de elétrons secundários e a composição química das microrregiões das amostras foram analisadas por EDS (Espectroscopia de Energia Dispersiva) com detector de raios X. Para a caracterização pelo método Brunauer-Emmett-Teller (BET), a amostra foi previamente seca em estufa por 24 horas à $100^{\circ} \mathrm{C}$, e posteriormente submetidas a um pré tratamento a $250^{\circ} \mathrm{C}$ por 4 horas, sob vácuo para retirar toda umidade e espécies adsorvidas da superfície do material. Em seguida, foram caracterizadas através de isotermas de adsorção/dessorção de $\mathrm{N}_{2}$.

A concentração de pesticida das amostras foi determinada por Cromatografia Líquida de Alta Performance (CLAE). Após a realização dos ensaios, as amostras coletadas foram filtradas em membranas de $0,45 \mu \mathrm{m}$ e $0,22 \mu \mathrm{m}$, e injetadas no cromatógrafo. Inicialmente, foi construída a curva de calibração, no Cromatógrafo Shimadzu ${ }^{\circledR}$ Prominence, para o padrão analítico atrazina. Para isto, foi preparada uma solução estoque de atrazina de $500 \mathrm{mg} \mathrm{L}^{-1}$, e a partir desta foram realizadas 
diluições, variando as concentrações de $0,05 \mathrm{mg} \mathrm{L}^{-1}$ à $10 \mathrm{mg} \mathrm{L}^{-1}$. Logo após, as amostras foram encaminhadas ao cromatógrafo.

As leituras da concentração de atrazina foram realizadas no Cromatógrafo Shimadzu® Prominence sob as condições de uso: Coluna C-18 (150mm x 4,6 mm), fase móvel acetonitrila/água (50:50, v/v), detector UV - $223 \mathrm{~nm}$, fluxo contínuo de 1 $\mathrm{mL} \min ^{-1}$, temperatura do forno de $35^{\circ} \mathrm{C}$, corrida de 6 minutos e volume de injeção de $20 \mu \mathrm{L}$ (Zheng et al., 2010), adaptado.

Para estimar os parâmetros do movimento de água e solutos em determinados solos, a partir de dados de curvas de eluição foi utilizado o programa DISP v1.1d, com os valores de entrada: teor de água $\left(\mathrm{cm}^{3} \mathrm{~cm}^{-3}\right)$, densidade do solo $\left(\mathrm{g} \mathrm{cm}^{-3}\right)$, fluxo $\left(\mathrm{cm} \mathrm{h}^{-1}\right)$, altura da coluna $(\mathrm{cm})$, Ci e Co (concentração inicial e a final encontradas), pulso (tempo de aplicação). Os parâmetros de transporte estimados pelo DISP foram: número de Peclet (Pe), fator de retardamento (R), dispersividade molecular (D) e difusividade do soluto em água $\left(\mathrm{D}^{\mathrm{w}}\right)$. A partir da determinação destes parâmetros foi possível rodar o programa para a simulação do transporte de solutos no solo.

\section{Resultados e Discussão}

\subsection{Caracterização do biocarvão}

A partir das propriedades físico-químicas do biocarvão verificou-se que o biocarvão de palha de milho apresentou área superficial relativamente alta (Tabela 1). Em estudos realizados por Zhang et al. (2013) é possível concluir que o valor da área superficial especifica está associado à matéria-prima de produção e alta área superficial específica resulta em maiores capacidades de adsorção (Khorram et al., 2016). Dessa forma, o biocarvão em estudo apresenta potencial para estudos adsortivos.

Tabela 1. Propriedades físico-químicas do biocarvão de palha de milho.

\begin{tabular}{lc}
\hline Área Superficial Específica $\mathrm{BET}\left(\mathrm{m}^{2} \mathrm{~g}^{-1}\right)$ & 388,054 \\
Diâmetro médio dos poros $(\AA)$ & 36,24 \\
Volume de poros $\left(\mathrm{cm}^{3} \mathrm{~g}^{-1}\right)$ & 0,016 \\
Tamanho médio de poros $(\AA)$ & 18,12 \\
Volume total de poros $\left(\mathrm{cm}^{3} \mathrm{~g}^{-1}\right)$ & 0,01619 \\
Volume de microporos $\left(\mathrm{cm}^{3} \mathrm{~g}^{-1}\right)$ & 0,1800 \\
$\mathrm{O}(\%)$ & 15,23 \\
$\mathrm{Si}(\%)$ & 0,26 \\
$\mathrm{P}(\%)$ & 4,08 \\
$\mathrm{O} / \mathrm{C}$ & 0,19 \\
\hline
\end{tabular}

Fonte: Autores (2021).

A porosidade do biocarvão tem forte efeito sobre a adsorção da atrazina, e estes poros podem ser classificados em três classes de tamanho, baseado no diâmetro: macroporos ( $>500$ Â), mesoporos $(20 \hat{A}<$ diâmetro $<500$ Â) e microporos $(<20 \hat{A})$ (IUPAC, 1985). O biocarvão apresenta parcela significativa de mesoporos, mas também com considerável presença de microporos, classificado então como micro-mesoporoso. Contudo, esta classificação não exclui a presença de macroporos. Em biocarvão de madeira, Xiao e Pignatello (2015) sugeriram que os mesoporos são importantes para facilitar a difusão da atrazina nas redes de poros. Nas micrografias obtidas por Microscopia Eletrônica de Varredura (MEV) (Figura 1) é possível verificar o início da formação dos poros e alguns já formados. As imagens mostram uma tendência mais evidente dos mesoporos, pouca 
evidência de micro. Apenas na Figura 1c é possível ver um poro tendendo a micro. Entretanto, o MEV capta imagens em uma porção de amostra muito pequena, podendo não ter aparecido nessa amostragem.

Figura 1. Imagens de Microscopia Eletrônica de Varredura a 7.000X (A, B) e 10.000 kx (C) de biocarvão de palha de milho.

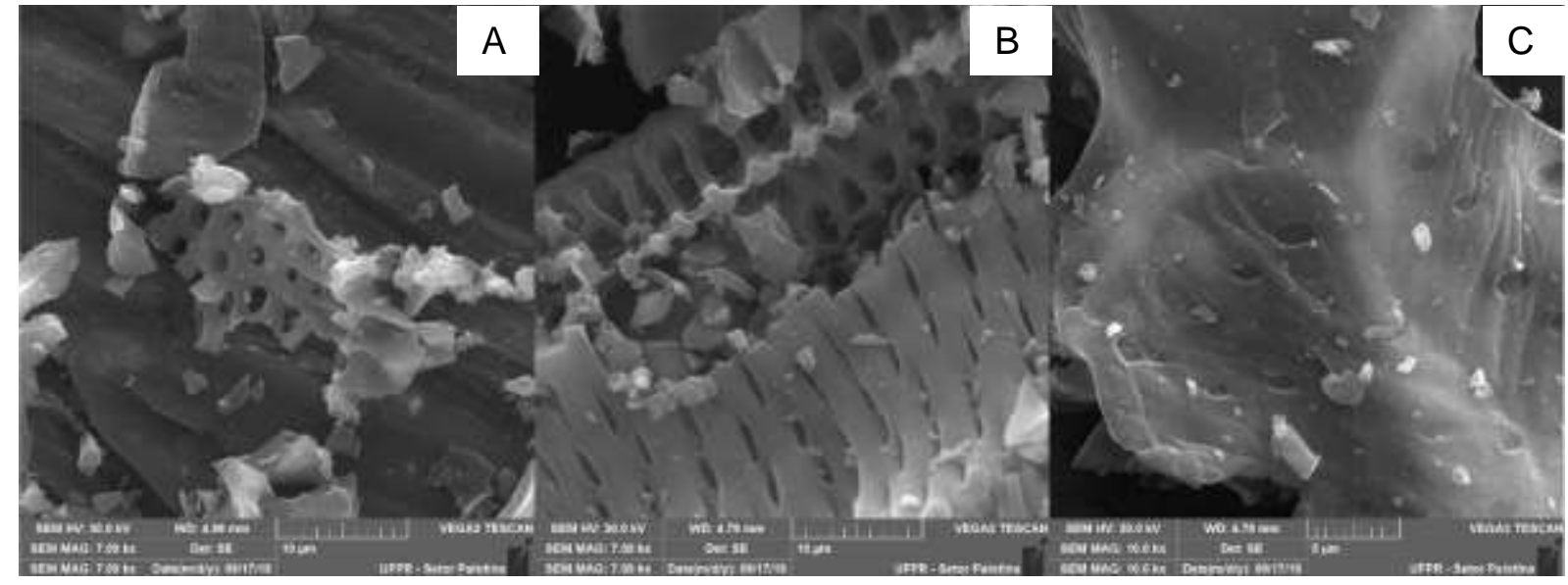

Fonte: Autores (2021).

Com base na isoterma de fisissorção de $\mathrm{N}_{2}$ (Figura 2), o biocarvão de palha de milho em estudo pode ser classificado como Tipo I(b) com histerese do tipo H4. A isoterma do tipo I(b) é associada com materiais com uma distribuição de tamanhos de poros mais ampla, incluindo microporos mais largos e mesoporos mais estreitos $(<2,5 \mathrm{~nm})$. A classificação da IUPAC para isotermas de adsorção define que histerese do tipo $\mathrm{H} 4$ é frequentemente encontrada em carvão ativado micro-mesoporoso (Thommes et al., 2015). A pressão média de histerese (P/P0 < 0,9880) é um indicativo que o material apresenta microporos e mesoporos em sua estrutura. Estes resultados estão em concordância com os parâmetros texturais observados (Tabela 1), em que, foi observado alta contribuição de microporos $\left(\right.$ Vmic $\left.=0,1800 \mathrm{~cm}^{3} \mathrm{~g}^{-1}\right)$.

Figura 2. Isoterma de adsorção-dessorção de $\mathrm{N}_{2}$ para o biocarvão de palha de milho.

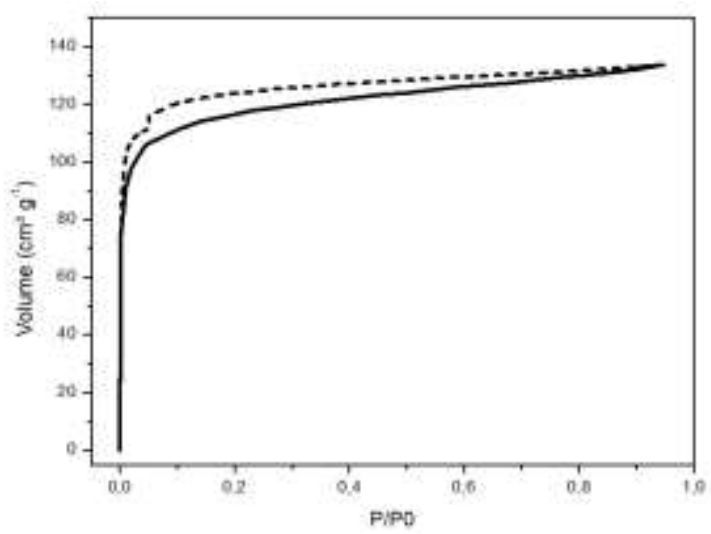

Fonte: Autores.

\subsection{Transporte de atrazina em colunas de solo indeformadas e deformadas}

As curvas de eluição de atrazina no solo registraram a capacidade que o solo estudado teve para armazenar o pesticida. Na Figura 3 é possível observar os valores obtidos dos dados experimentais nas colunas de solo indeformadas. Verificou-se que as colunas de solo sem biocarvão apresentaram maior transporte de atrazina, uma vez que os picos do pesticida se encontram em concentrações maiores se comparadas às colunas de solo com o biocarvão. A permeabilidade do 
solo é um atributo reflexo da textura e da estrutura do solo, ou seja, quanto mais estruturado o solo for, maior a permeabilidade e maior será a facilidade de movimento do pesticida através do perfil do solo (Flury, 1996). Dessa forma, de acordo com os resultados obtidos, a utilização do biocarvão em solo pode diminuir a lixiviação da atrazina e em consequência reduzir o risco de contaminação (Deng et al., 2017).

Figura 3. Concentrações relativas de atrazina em coluna de solo indeformada em 5 volumes de poros.

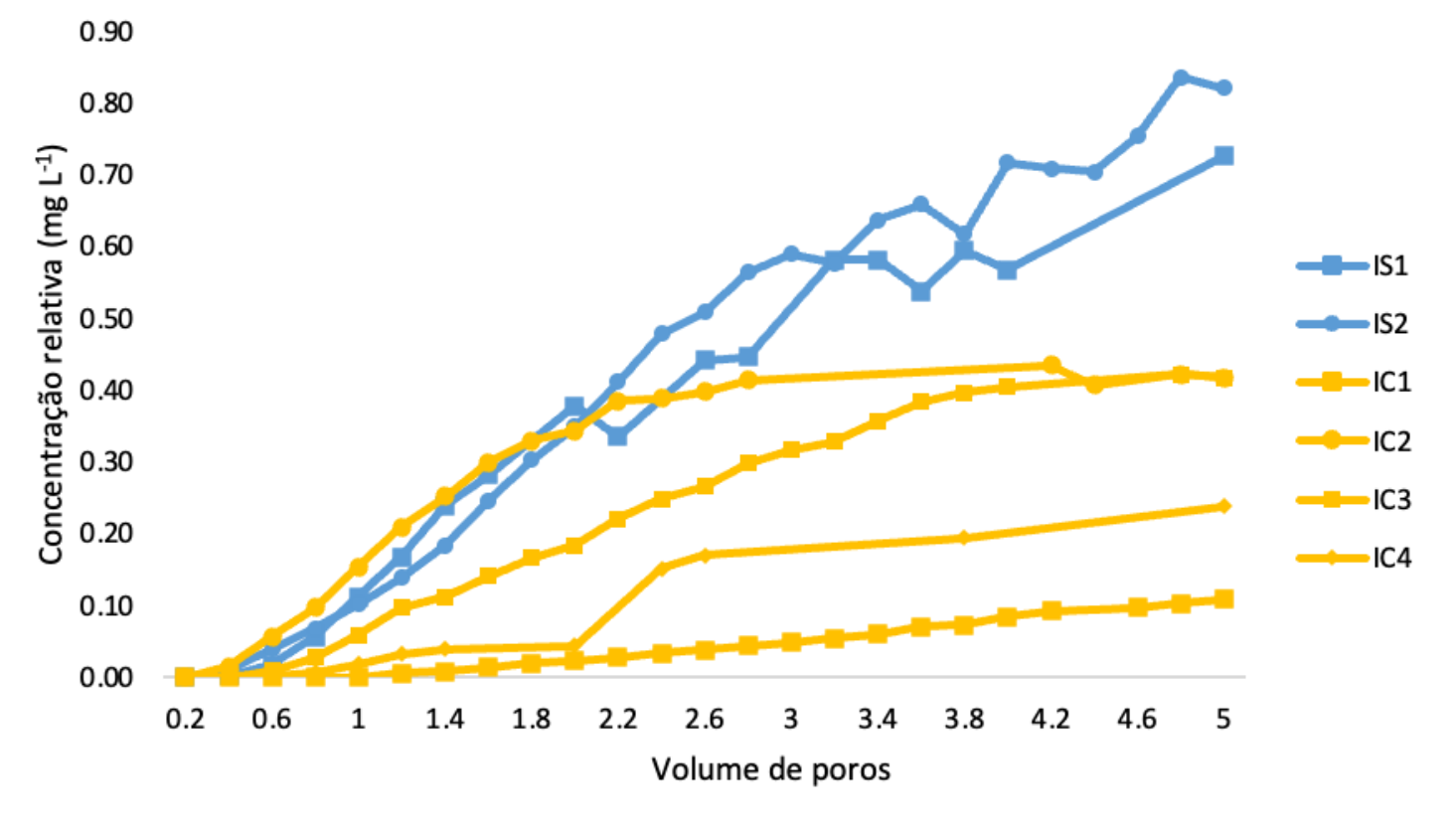

IS: Indeformada sem biocarvão; IC: Indeformada com biocarvão. Fonte: Autores.

$\mathrm{Na}$ análise de pesticida adsorvido em colunas de solo deformadas verificou-se que as colunas de solo sem biocarvão, assim como nas colunas indeformadas, apresentaram menor adsorção de atrazina, se comparadas as com o biocarvão (Figura 4). Ainda, as colunas com biocarvão apresentaram concentrações menores na quantidade de volume de poros analisados.

Figura 4. Concentrações relativas de atrazina em coluna de solo deformada em 5 volumes de poros.

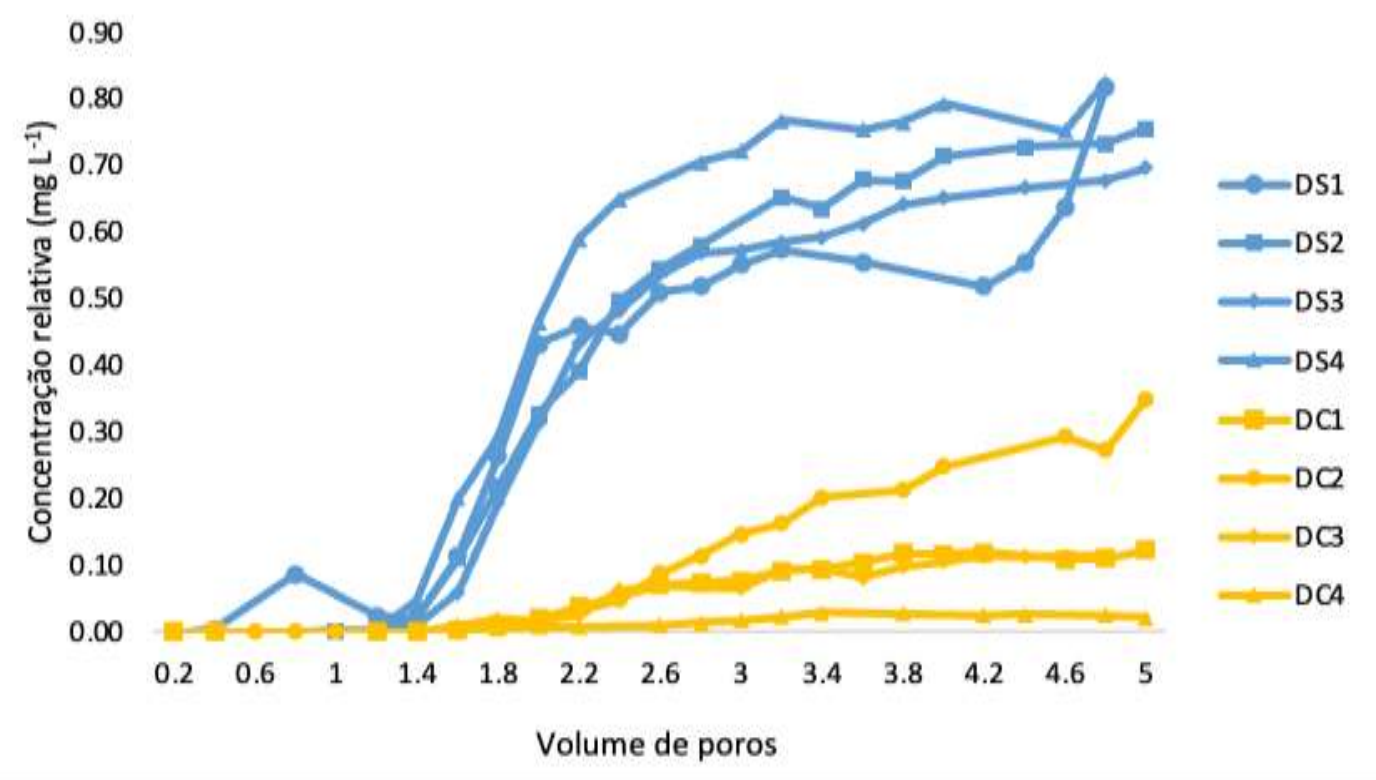

DS: Deformada sem biocarvão; DC: Deformada com biocarvão. Fonte: Autores. 
O biocarvão causou variação no comportamento da atrazina, tanto no solo deformado quanto no indeformado, mostrando que interfere diretamente no transporte do pesticida, reduzindo a concentração lixiviada na faixa de 50 a $95 \%$ (se analisar a concentração média do volume de poros 5 no gráfico sem biocarvão $\left(0,8 \mathrm{mg} \mathrm{L}^{-1}\right)$ e com biocarvão $\left(0,03\right.$ a $\left.0,4 \mathrm{mg} \mathrm{L}^{-1}\right)$.

$\mathrm{Na}$ Figura 5 verifica-se o efeito do biocarvão de acordo com a metodologia utilizada para montagem das colunas. Sendo possível constatar que nas colunas indeformadas e com a presença do biocarvão o tempo de retenção da solução de atrazina dentro da coluna foi relativamente menor, se comprado às colunas deformadas. Enquanto que, na ausência do biocarvão, as duas colunas apresentaram comportamento semelhante, variando, consideravelmente pouco, o tempo de retenção dentro da coluna. Foi possível constatar ainda, que as colunas com biocarvão apresentaram concentrações menores do que as sem biocarvão, no volume de poros analisados.

Figura 5. Concentrações relativas de atrazina em coluna de solo indeformada e deformada em 5 volumes de poros. Colunas de solo com biocarvão (a) e colunas de solo sem biocarvão (b)

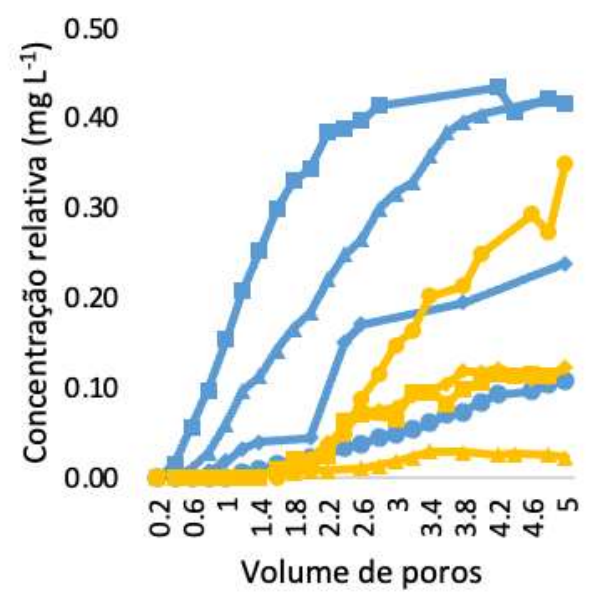

(a)

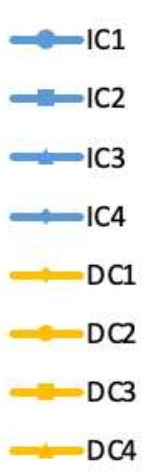

DC4

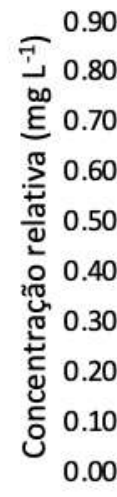

.00

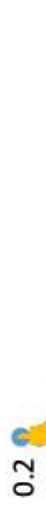

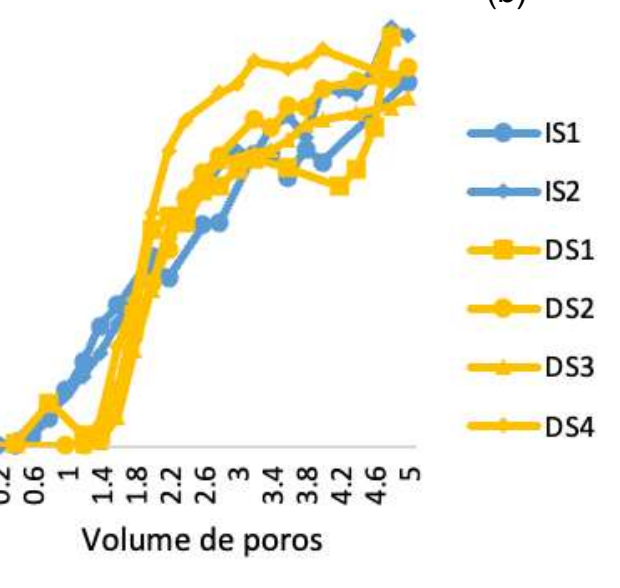

Fonte: Autores.

Em experimentos realizados por Rossi et al. (2007) comparando colunas de solo deformadas e indeformadas, foi possível constatar que na deformada os resultados não se apresentaram representativos para os parâmetros de transporte analisados, por isso consideram como situação ideal o solo estruturado, além deste representar mais fielmente as condições naturais. Já Sampaio et al. (2010) constataram que a metodologia de colunas de solo indeformadas não se mostrou adequada, já que os resultados satisfatórios se apresentaram em colunas de solo deformadas. Ainda concluem que o problema é devido, possivelmente, à compactação do solo durante a amostragem e à dificuldade no bloqueio de caminhos preferenciais ao longo de toda a coluna.

Motivo que explica, é que as propriedades hidráulicas do solo se modificam com as características das partículas, com o seu conteúdo de água e com sua geometria porosa. Além disso, as mudanças significativas na condutividade hidráulica, no fluxo de água e nutrientes, são previstas entre irrigações, mesmo que o conteúdo de água seja mantido dentro do que é denominado água facilmente disponível (Barreto et al., 2012). A condutividade hidráulica de um solo é a velocidade com que um líquido atravessa um meio poroso (Reichardt \& Timm, 2004) e varia com o potencial de água no seu interior, ocorrendo elevação acentuada de acordo com a quantidade de água (Caron \& Elrick, 2005). Desta forma, quanto maior a condutividade hidráulica, mais rápido a solução passa pela coluna de solo.

A maior condutividade na superfície do solo pode representar aumento nas perdas do pesticida por lixiviação, assim, quando a quantidade de água excede a capacidade de infiltração do solo, começam as perdas por escoamento superficial. Fato 
este que não ocorreu, devido à condução do experimento. A menor condutividade na superfície aumenta o tempo de contato da atrazina com o solo, favorecendo o processo de adsorção e redução das perdas por lixiviação (Correia et al., 2007).

\subsection{Transporte de atrazina estimado pelo DISP v1.1d}

Os parâmetros de transporte de atrazina no solo foram estimados pelo programa DISP v1.1d, em que foram fornecidos os valores para montagem das curvas de eluição, em até 5 volumes de poro. Os valores estimados dos parâmetros de transporte de solutos são apresentados na Tabela 2 .

Tabela 2. Parâmetros de transporte de solutos no solo fornecidos pelo DISP. Pe (número de péclet); R (fator de retardamento); $\mathrm{D}$ (dispersividade molecular); $\mathrm{D}^{\mathrm{w}}$ (difusividade do soluto em água); $\mathrm{R}^{2}$ (coeficiente de determinação)

\begin{tabular}{lccccc}
\hline Colunas & $\mathrm{Pe}$ & $\mathrm{R}$ & $\mathrm{D}(\mathrm{cm})$ & $\mathrm{D}^{\mathrm{w}}\left(\mathrm{cm}^{2} \mathrm{~h}^{-1}\right)$ & $\mathrm{R}^{2}$ \\
\hline IND SEM 1 & 1,39 & 4,997 & 21,583 & 63,3813 & 0,985 \\
IND SEM 2 & 2,79 & 3,525 & 10,753 & 63,0466 & 0,992 \\
IND COM 1 & 1,45 & 13,373 & 20,690 & 86,0000 & 0,819 \\
IND COM 2 & 2,80 & 15,102 & 10,714 & 18,7143 & 0,793 \\
IND COM 3 & 0,51 & 10,915 & 58,824 & 106,6667 & 0,787 \\
IND COM 4 & 0,70 & 12,690 & 42,857 & 226,1429 & 0,986 \\
\hline DEF SEM 1 & 2,08 & 4,413 & 14,423 & 103,0288 & 0,882 \\
DEF SEM 2 & 5,88 & 2,699 & 5,102 & 53,4864 & 0,950 \\
DEF SEM 3 & 4,52 & 3,364 & 6,637 & 43,8717 & 0,967 \\
DEF SEM 4 & 3,46 & 3,768 & 8,671 & 66,1272 & 0,943 \\
DEF COM 1 & 5,30 & 15,417 & 5,660 & 43,2453 & 0,032 \\
DEF COM 2 & 350,10 & 2,693 & 0,086 & 1,2028 & 0,047 \\
DEF COM 3 & 2,18 & 15,478 & 13,761 & 130,8257 & 0,699 \\
DEF COM 4 & 2,44 & 14,662 & 12,295 & 166,4344 & 0,755 \\
\hline
\end{tabular}

Fonte: Autores (2021).

Os aspectos gerais dos mecanismos físicos de transporte e retenção de contaminantes em meios porosos saturados podem ser divididos em transporte por advecção ou difusão. Desta forma, o tipo de transporte dominante de acordo com o número de Peclet pode ser descrito entre transição e advecção. Ou seja, o transporte de contaminantes é devido ao processo de fluxo de água no solo, ou os solutos presentes na água se movimentam com velocidade igual à velocidade da mesma, sem alterar a sua concentração na solução. Os resultados encontrados em uma das colunas com solo deformado com biocarvão se apresentaram fora dos padrões encontrados nas outras colunas, e isto se deve a caminhos preferenciais no interior da coluna.

$\mathrm{O}$ fator de retardamento representa a razão entre a concentração dos solutos na fase sólida e líquida, e quando o R aumenta, a adsorção também aumenta (Santos, 2014). Dessa forma, nota-se que o biocarvão reteve maiores concentrações de atrazina. Correia et al. (2010) concluíram que o processo de retenção de atrazina está diretamente ligado à característica estrutural do solo e os baixos valores de R confirmam o potencial de deslocamento do pesticida mesmo em solos de baixa infiltração. E devido à irregularidade das curvas características de transporte nos solos, não determinaram o coeficiente de dispersão deste herbicida. Rossi et al. (2007) constataram em seus experimentos que as amostras deformadas apresentaram fator de retardamento maior, comparadas aos mesmos solos, às amostras indeformadas. 
De acordo com os valores de dispersividade molecular (D) encontrados, foi possível detectar que a adição de biocarvão dispersa a atrazina no solo, atrasando assim, a sua lixiviação no solo. E a difusão em água, demonstrada pela difusividade do soluto $\left(\mathrm{D}^{\mathrm{w}}\right)$, é maior nas colunas que tiveram biocarvão adicionado, já que o produto equilibra as moléculas de atrazina na água. Desta forma, quando os valores são baixos, conclui-se que a atrazina fica concentrada em alguns pontos, o que facilita a lixiviação concentrada da solução.

Os valores do coeficiente de determinação $\left(R^{2}\right)$ expressam quanto da variação total dos dados é explicada pela equação de regressão. Desta forma, os valores encontrados afirmam que o experimento conduzido em colunas de solo deformadas não apresentou resultados satisfatórios, uma vez que, valores mais próximos de 1 indicam alta aderência ao modelo testado. Rossi et al. (2007) constataram que os erros evidenciaram efeito da forma de preenchimento das colunas, já que se altera a densidade do solo em relação às condições naturais. Ademais, ao deformar a coluna, o solo desestruturado favorece o entupimento dos poros, resultando em maior resistência à penetração e maior teor de retenção de água (Grego \& Vieira, 2005; Correia et al., 2010).

\section{Conclusão}

A estrutura construída e automatização facilitaram a aplicação da solução de atrazina nas colunas de solo, e posterior coleta das amostras.

A metodologia utilizada para construção das colunas de solo deformadas necessita ser revisada, uma vez que os resultados provenientes delas não são estatisticamente aceitos, já que quanto mais próximos a 1 , os valores indicam alta aderência ao modelo testado.

O biocarvão, na quantidade estudada de 5 ton ha ${ }^{-1}$ se mostrou eficiente quanto à adsorção de atrazina, tanto nas colunas deformadas, quanto nas indeformadas. Porém, para as colunas deformadas, as concentrações de atrazina percolada apareceram após um tempo maior de retenção na coluna, sendo necessário um estudo com uma quantidade de volume de poros maior.

A eficiência do programa DISP v1.1d foi satisfatória, uma vez que, auxiliou no ajuste dos resultados de eluição, simulando os parâmetros do movimento da atrazina no solo, facilitando a visualização da eficiência do transporte de solutos no solo.

\section{Agradecimentos}

Agradecemos a CAPES pelo apoio e concessão de bolsa de estudos e ao CNPq pelo financiamento do projeto.

\section{Referências}

Adams, M., Crawford, J., Field, D., Henakaarchchi, N., Jenkins, M., McBratney, A. \& Wheeler, J. (2011). Managing the soil-plant system to mitigate atmospheric $\mathrm{CO}_{2}$. Documento de discussão para o Soil Carbon Sequestration Summit. O Centro de Estudos dos Estados Unidos da Universidade de Sydney.

Alonso, D. G., Koskinen, W. C., Oliveira Jr., R. S., Constantin, J. \& Mislankar, S. (2011). Sorption-desorption of indaziflam in selected agricultural soils. Journal of Agricultural and Food Chemistry, 59(24), 13096- 13101.

Barreto, C. V. G., Testezlaf, R. \& Salvador, C. A. (2012). Ascensão Capilar De Água Em Substratos De Coco E De Pinus. Bragantia, 71, $385-399$.

Barreto, F. M. S. (2006) Contaminação da Água Subterrânea por Pesticidas e Nitrato no Município de Tianguá, Ceará. Tese. Universidade Federal Do Ceará. - Ufc, Fortaleza.

Borras, N., Oliver, R., Arias, C. \& Crillas. E. (2010). Degradation of Atrazine by Electrochemical Advanced Oxidation Process Using a Boron-Doped Diamond Anode. The Journal of Physical Chemistry. 114, 6613-6621.

Cabrera, A., Cox, L., Spokas, K., Celis, R., Hermosin, C., Cornejo, J. \& Koskinen, W.C. (2011). Comparative Sorption And Leaching Study Of The Herbicides Fluometuron And 4-Chloro-2-Methylphenoxyacetic Acid (Mcpa) In A Soil Amended With Biochars And Other Sorbents. Journal Of Agricultural And Food Chemistry. 59, 12550-12560. 
Canuto, T. G., Gama, A. F., Barreto, F. M. de S. \& Alencar Neto, M. da F. (2010). Estimativa Do Risco Potencial De Contaminação Por Pesticidas De Águas Superficiais E Subterrâneas Do Município De Tianguá-Ce, Com Aplicação Do Método De Goss E Índice De Gus. Águas Subterrâneas, https://aguassubterraneas.abas.org/asubterraneas/article/view/23083

Cao, X., Ma, L., Gao, B. \& Harris, W. (2009). Dairy-Manure Derived Biochar Effectively Sorbs Lead and Atrazine. Environmental Science \& Technology. 43(9), 3285-3291.

Cao, X., Ma, L., Liang, Y., Gao, B. \& Harris, W. (2011). Simultaneous Immobilization of Lead and Atrazine in Contaminated Soils Using Dairy-Manure Biochar. Environmental Science \& Technology. 45(11), 4884-4889.

Caron, J. \& Elrick, D. (2005). Measuring The Unsaturated Hydraulic Conductivity of Growing Media with A Tension Disc. Soil Science Society American Journal. 69, 783-793.

Correia, F. V., Langenbach, T. \& Campos, T. M. (2010). Avaliação Do Transporte De Atrazina Em Solos Sob Diferentes Condições De Manejo Agrícola. Revista Brasileira De Ciência Do Solo. 34(2), 525-534.

Correia, F. V., Mercante, F. M., Fabricio, S. C., Campos, T. M. De, Vargas Junior, E. A. \& Langenbach, T. (2007). Infiltração de Atrazina em Latossolo Submetido aos Sistemas de Plantio Direto e Convencional. Pesquisa Agropecuária Brasileira. 42(11), 1617-1625.

Deng, H., Feng, D., He, J., Li, F., Yu, H. \& Ge, C. (2017) Influence of Biochar Amendments to Soil on The Mobility of Atrazineusing Sorption-Desorption and Soil Thin-Layer Chromatography. Ecological Engineering. 99, 381-390.

Delwiche, K. B., Lehmann, B. J. \& Walter, M. T. (2014). Atrazine Leaching from Biochar-Amended Soils. Chemosphere. 95, 346-352.

Empresa Brasileira De Pesquisa Agropecuária - EMBRAPA. (2017). Biocarvão Feito Com Resíduos É Testado Como Condicionador De Solo. Editor Técnico: Gabriel Faria. https://www.embrapa.br/busca-de-noticias/-/noticia/28595289/biocarvao-feito-com-residuos-e-testado-como-condicionador-de-solo.

Farias, W. M., Pacheco, L. C. P. S., Figueiredo, C. C. \& Damin, V. (2016). Sorção Do Oxyfluorfen Em Solo Com Aplicação De Biochar De Lodo De Esgoto Determinada Por Bioensaio. Anais do XXXV Congresso Brasileiro De Ciência Do Solo.

Ferreira, P. A., Garcia, G. De O., Matos, A. T. de, Ruiz, H. A. \& Borges Junior, J. C. F. (2006). Transporte No Solo De Solutos Presentes Na Água Residuária De Café Conilon. Acta Scientiarum. Agronomy. 28(1), 29-35.

Figueredo, N. A., Costa, L. M., Melo, L. C. A., Siebeneichlerd, E. A. \& Tronto, J. (2017). Characterization of Biochars from Different Sources and Evaluation of Release of Nutrients and Contaminants. Revista Ciência Agronômica. 48(3), 395-403.

Flury, M. (1996). Experimental Evidence of Transport of Pesticides Through Field Soils: A Review. Journal of Environmental Quality. 25(1), 25-45.

Grego, C. R. \& Vieira, S. R. (2005). Variabilidade Espacial De Propriedades Físicas Do Solo Em Uma Parcela Experimental. Revista Brasileira De Ciência Do Solo. 29, 169-177.

International Union of Pure and Applied Chemistry - IUPAC. (1985). Reporting Physisorption Data for Gas/Solid Systems with Special Reference to the Determination of Surface Area and Porosity. Pure and Applied Chemistry. 57, 603-619.

Khorram, M.S., Zhang, Q., Lin, D., Zheng, Y., Fang, H. \& Yu, Y. (2016). Biochar: A Review of its Impact on Pesticide Behavior in Soil Environments and its Potential Applications. Journal Of Environmental Sciences. 44, 269-279.

Leach, M., Fairhead, J. \& Fraser, J. (2012). Green Grabs and Biochar: Revaluing African Soils and Farming In The New Carbon Economy. Journal of Peasant Studies. 39, 285-307.

Oliveira Jr., R. S. (2002). Conceitos Importantes No Estudo Do Comportamento De Herbicidas No Solo. Boletim Informativo, Sociedade Brasileira De Ciência Do Solo. 27(2), 9-12.

Reichardt, K. \& Timm, L. C. (2004). Solo, Planta e Atmosfera: Conceitos, Processos e Aplicações. Barueri: Manole.

Rezende, E. I. P., Angelo, L. C., Dos Santos, S. S. \& Mangrich, A. S. (2011). Biocarvão (Biochar) e Sequestro de Carbon. Revista Virtual de Química. 3(5), 426-433.

Rojas, R., Morillo, J., Usero, J., Vanderlinden, E. \& El Bakouri, H. (2015). Adsorption Study of Low-Cost and Locally Available Organic Substances and a Soil to Remove Pesticides from Aqueous Solutions. Jounal Hydrology. 520, 461-472.

Rossi, P., Miranda, J. H. \& Duarte, S. N. (2007). Curvas de Distribuição de Efluentes do Íon Nitrato em Amostras de Solo Deformadas e Indeformadas. Engenharia Agrícola. 27(3), 675-682.

Sampaio, S. C., Caovilla, F. A., Opazo, M. A. U., Nóbrega, L. H. P., Suszek, M. \& Smanhotto, A. (2010). Lixiviação de Íons em Colunas de Solo Deformado e Indeformado. Engenharia Agrícola. 30(1), 150-159.

Santos, R. S. S. (2014). Simulação da Dinâmica do Íon Potássio pelo Modelo Hydrus-1d em Condiçães de Solo Salino. Tese. Universidade de São Paulo, Piracicaba.

Tatarková, V., Hiller, E. \& Vaculík, M. (2013). Impact of Wheat Straw Biochar Addition to Soil on the Sorption, Leaching, Dissipation of The Herbicide (4Chloro-2-Methylphenoxy) Acetic Acid and The Growth of Sunflower (Helianthus Annuus L.). Ecotoxicology and Environmental Safety. 92, $215-221$.

Thommes, M., Kaneko, K., Neimark, A. V., Olivier, J. P., Rodriguez-Reinoso, F., Rouquerol, J. \& Sing, K. S. (2015). Physisorption of gases, with special reference to the evaluation of surface area and pore size distribution (IUPAC Technical Report). Pure and Applied Chemistry. 87(9-10), 1051-1069. 
Research, Society and Development, v. 10, n. 15, e396101522826, 2021

(CC BY 4.0) | ISSN 2525-3409 | DOI: http://dx.doi.org/10.33448/rsd-v10i15.22826

Xiao, F. \& Pignatello, J. J. (2015). Interactions of Triazine Herbicides with Biochar: Steric and Electronic Effects. Water Research. 80, 179-188.

Zhang, P. Sun, H., Yu, L. \& Sun, T. (2013). Adsorption and Catalytic Hydrolysis of Carbaryl and Atrazine on Pig Manure-Derived Biochars: Impact of Structural Properties of Biochars. Journal of Hazardous Material. 244, 217. 